STUDI

FRANCESI

\section{Studi Francesi}

Rivista quadrimestrale fondata da Franco Simone

194 (LXV | II) | 2021

Baudelaire et son cénacle

\title{
Tristan, chevalier de la Table Ronde. Roman imprimé en 1489 pour Anthoine Vérard, Édition de M.B. WINN
}

\section{Paola Cifarelli}

\section{(2) OpenEdition}

\section{Journals}

\section{Édition électronique}

URL : https://journals.openedition.org/studifrancesi/44734

DOI : 10.4000/studifrancesi.44734

ISSN : 2427-5856

Éditeur

Rosenberg \& Sellier

\section{Édition imprimée}

Date de publication : 1 août 2021

Pagination : $351-352$

ISSN : 0039-2944

\section{Référence électronique}

Paola Cifarelli, «Tristan, chevalier de la Table Ronde. Roman imprimé en 1489 pour Anthoine Vérard,

Édition de M.B. winn », Studi Francesi [En ligne], 194 (LXV | II) | 2021, mis en ligne le 06 septembre 2021, consulté le 15 octobre 2022. URL : http://journals.openedition.org/studifrancesi/44734 ; DOI : https:// doi.org/10.4000/studifrancesi.44734

Ce document a été généré automatiquement le 15 octobre 2022

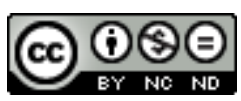

Creative Commons - Attribution - Pas d'Utilisation Commerciale - Pas de Modification 4.0 International - CC BY-NC-ND 4.0

https://creativecommons.org/licenses/by-nc-nd/4.0/ 


\title{
Tristan, chevalier de la Table Ronde. Roman imprimé en 1489 pour Anthoine Vérard, Édition de M.B. WINN
}

\author{
Paola Cifarelli
}

\section{RÉFÉRENCE}

Tristan, chevalier de la Table Ronde. Roman imprimé en 1489 pour Anthoine Vérard, Édition de M.B. winN, Paris, Classiques Garnier, 2020, «Textes littéraires du Moyen Âge» 53, série Le Moyen Âge dans les imprimés 1, 2 vols, 1277 pp.

1 M.B. Winn, grande spécialiste de la production de l'éditeur-libraire Anthoine Vérard et auteur de l'un des ouvrages les plus importants pour l'étude de l'activité de ce dernier, fournit ici l'édition du Tristan en prose d'après l'editio princeps imprimée pour Vérard par Jehan Le Bourgeois, à Rouen en 1489. Ce volume imposant inaugure, au sein de la collection bien connue des «Textes Littéraires du Moyen Âge», une série dirigée par A. Schoysman, consacrée aux imprimés ayant diffusé la culture médiévale au tournant du Xvie siècle.

2 Le choix d'éditer l'imprimé se justifie non seulement pour l'intérêt documentaire de ce texte, qui permit de faire connaître à un public plus vaste un texte voué à un grand succès, mais aussi pour l'originalité de la version transmise par cet incunable; en effet, un seul des 80 manuscrits qui nous l'ont conservé (Paris, BnF, fr. 103, proche de l'incunable sans en être la source) contient cette histoire abrégée de Tristan et de la belle Yseut, pourvue d'un nouveau dénouement touchant les circonstances de la mort du héros éponyme. L'édition critique se fonde sur l'exemplaire Paris, BnF, Rés. Y2 57-58, l'un des huit exemplaires complets connus actuellement; elle fait suite à la publication du fac-similé de l'incunable, en 1978, par Cédric Pickford, qui avait déjà fourni un certain nombre de données sur la tradition textuelle. La grande compétence de M.B.W. dans le domaine de l'histoire du livre fait de la première partie de 
l'introduction un véritable modèle de description de l'imprimé; on y trouvera, entre autres, l'indication des caractéristiques propres à chacun des exemplaires connus, y compris les copies fragmentaires, ainsi que plusieurs éléments significatifs pour l'identification des stratégies éditoriales, voire publicitaires, qui sous-tendent la publication du Tristan en prose: la structure du colophon et la répartition du texte en deux volumes en sont deux exemples. La reconstruction précise des circonstances de l'édition et de la nature de la collaboration entre Jehan le Bourgeois et Vérard est un autre élément particulièrement intéressant de cette introduction, qui situe le Tristan dans le cadre des débuts de la carrière du libraire-éditeur parisien. L'étude de la diffusion de l'ouvrage, réimprimé six fois jusqu'en 1533 tant par Vérard que par d'autres imprimeurs parisiens, est tout aussi précise et elle permet d'apprécier les modifications introduites successivement à plusieurs niveaux: introduction d'illustrations, exécution de copies sur vélin enluminées, utilisation de caractères typographiques différents, réduction progressive du format, impression des pièces lyriques sur des lignes individuelles, renouvellement de la page de titre en 1533, modernisation des graphies et du lexique. L'analyse des rapports de l'editio princeps avec le ms. fr. 103, enluminé par le Maître de l'Échevinage de Rouen et appartenu au célèbre bibliophile Louis de la Gruthuyse, sert d'introduction à l'étude du texte transmis par l'incunable de 1489; longtemps négligé en raison du jugement fortement critique de Joseph Bédier, le texte en prose a pourtant joui d'études importantes à partir de 1975. Quatre versions ont été identifiées, dont la n. IV, qui constitue la base de celle transmise par le ms. fr. 103; la proximité de celle-ci avec les versions en vers concerne surtout le dénouement. La comparaison de l'imprimé avec le manuscrit permet de corriger les conclusions des études précédentes et de souligner l'originalité de l'imprimé, qui contient non seulement un texte abrégé, mais aussi des amplifications et des modifications dans l'agencement de quelques épisodes qui lui sont propres.

3 L'étude de la langue du texte met en évidence les faits linguistiques caractérisant ce texte, qui présente quelques traits picards et un certain nombre d'archaïsmes tant dans le groupe du nom que dans la morphologie du verbe, malgré le souci de l'éditeur de réduire les picardismes et de rajeunir la langue. La ponctuation est elle aussi l'objet d'une description très précise, qui permet de recenser tous les éléments destinés à indiquer le rythme et la structuration syntaxique du texte.

Dans sa transcription interprétative, l'A. n'intervient que pour corriger les erreurs évidentes et les coquilles. L'exemplaire de base est contrôlé sur le volume conservé à Oxford, Bodleian Library, Douce 224 (0), venant de la même édition mais comportant quelques interventions sur le texte au cours du tirage de certains feuillets; les éditions plus tardives ont été utilisées très opportunément pour mieux comprendre certains passages et, dans quelques cas, pour les corriger, comme au l. II, ch. 28, par. 2 et 3. L'apparat en bas de page contient les variantes des éditions postérieures et les leçons qui ont fait l'objet d'une intervention de la part de l'éditrice. Les notes réunies à la fin de chaque volume concernent, comme il se doit, des observations philologiques et linguistiques à propos de certaines leçons particulières, des indications sur les variantes concernant la structuration de la matière en chapitres, des éclaircissements sur des passages problématiques, des explications sur les corrections apportées au texte de base, ainsi que des comparaisons avec d'autres versions de la prose ou d'autres textes susceptibles de fournir des précisions sur des détails de certains épisodes. Elles 
permettent toujours de justifier les choix de l'éditrice et elles contribuent efficacement à la compréhension du texte.

5 Le glossaire, riche et précis, est suivi d'un Index des noms propres, d'un Index des proverbes et expressions sentencieuses recensés d'après le mot-clé comportant l'indication de l'attestation éventuelle dans le Nouveau dictionnaire des locutions de G. Di Stefano et d'un répertoire des passages du texte fournissant des indications sur les mœurs et habitudes sociales évoqués. Sept Annexes concernent respectivement: la description des éditions postérieures du Tristan en prose, l'indication des feuillets détachés du Tristan de 1489, la transcription des prologues figurant dans la copie Paris, BnF, Rés. Y2 57 et dans le ms. fr. 103, la liste des rubriques des chapitres de l'édition de 1489, les concordances entre les chapitres de 1489 et les passages correspondants dans les autres versions de la prose, les chapitres ajoutés au t. II dans l'édition de $1496 \mathrm{ca}$, la liste des pièces en vers. La bibliographie clôt cette édition, qui fournira aux spécialistes un instrument fiable pour exploiter la richesse de ce texte. 